\title{
Equipamentos coletores para estomias: Percepção do usuário de um Centro
}

\section{Especializado em Reabilitação}

\author{
Collecting equipment for ostomies: User perception of a Specialized Rehabilitation Center \\ Equipo colector de ostomía: Percepción del usuario de un Centro de Rehabilitación Especializado
}

Recebido: 24/07/2021 | Revisado: 28/07/2021 | Aceito: 30/07/2021 | Publicado: 05/08/2021

\author{
Aliene Cristina dos Santos \\ ORCID: https://orcid.org/0000-0002-8443-3414 \\ Prefeitura Municipal de Promissão, Brasil \\ E-mail: aliene_crist@hotmail.com \\ Maria Helena Pinto \\ ORCID: https://orcid.org/0000-0003-0601-8511 \\ Faculdade de Medicina de São José do Rio Preto, Brasil \\ E-mail: helmarpi@gmail.com \\ Adriana Pelegrini dos Santos Pereira \\ ORCID: https://orcid.org/0000-0003-4237-365X \\ Faculdade de Medicina de São José do Rio Preto, Brasil \\ E-mail: adrianapelegrini@famerp.br \\ João Júnior Gomes \\ ORCID: https://orcid.org/0000-0003-4171-2752 \\ Centro Universitário São Camilo, Brasil \\ E-mail:jjenf@terra.com.br \\ Janderson Cleiton Aguiar \\ ORCID: https://orcid.org/0000-0002-6095-8689 \\ União das Faculdades dos Grandes Lagos, Brasil \\ E-mail: janderpoty@yahoo.com.br \\ Kellyngton Gomes da Silva \\ ORCID: https://orcid.org/0000-0001-8382-1637 \\ Universidade Paulista, Brasil \\ E-mail: kellyngtongomes1@gmail.com
}

\begin{abstract}
Resumo
O objetivo deste estudo foi caracterizar a população com estomia atendido no Centro Especializado de Reabilitação e verificar a percepção do usuário em relação a qualidade, segurança, quantidade e a frequência de troca dos equipamentos fornecidos pelo serviço. Trata-se de um estudo transversal realizado em um Serviço de Atenção ao Estomizado, do interior do estado de São Paulo, Brasil. Foram entrevistadas 126 pessoas com estomias intestinais, entre os meses de abril a junho de 2019, no dia agendado para a aquisição dos equipamentos. Entre os participantes do estudo, $62,90 \%$ eram do sexo masculino e $64,29 \%$ com estomias definitiva; $80,16 \%$ afirmaram estar totalmente satisfeitos com a qualidade do material recebido e $67,46 \%$ manifestaram total segurança com o uso do equipamento coletor; $92,08 \%$ afirmaram estar satisfeitos com as orientações recebidas pela equipe de saúde e materiais recebidos. Os testes estatísticos não evidenciaram dependência entre qualidade do equipamento coletor com as demais variáveis $(p>0,05)$. A maioria dos entrevistados relatou estar satisfeito com a qualidade do equipamento e o atendimento fornecido pelo serviço. Destaca-se a importância do enfermeiro estomaterapeuta, que é essencial no cuidado de pacientes com estomias, desde o período pré-operatório e acompanhamento no pós-operatório, com indicação do equipamento coletor adequado para alcance da reabilitação e desenvolvimento da autonomia da pessoa que possui uma estomia.
\end{abstract}

Palavras- chave: Percepção; Estomia; Assistência à saúde; Centros de reabilitação; Assistência ao paciente.

\begin{abstract}
The aim of this study was to characterize the population with ostomy treated at the Specialized Rehabilitation Center and to verify the user's perception of quality, safety, quantity and frequency of exchange of equipment provided by the service. This is a cross-sectional study carried out in an Ostomy Care Service in the interior of the state of São Paulo, Brazil. 126 people with intestinal ostomy were interviewed between the months of April and June 2019, on the day scheduled for the acquisition of the equipment. Among the study participants, $62.90 \%$ were male and $64.29 \%$ with permanent ostomies; $80.16 \%$ said they were totally satisfied with the quality of the material received and $67.46 \%$ expressed total security with the use of the collecting equipment; $92.08 \%$ said they were satisfied with the guidance received by the health team and materials received. Statistical tests did not show dependence between the quality of the collecting equipment and the other variables $(p>0.05)$. Most respondents reported being satisfied with the quality of the equipment and the care provided by the service. The importance of the stomatherapist nurse is highlighted, as it is
\end{abstract}


essential in the care of patients with ostomies, from the preoperative period and postoperative follow-up, with indication of the appropriate collecting equipment to achieve the rehabilitation and development of the autonomy of the person who has an ostomy.

Keywords: Perception; Ostomy; Delivery of health care; Rehabilitation centers; Patient care.

\section{Resumen}

El objetivo de este estudio fue caracterizar a la población con ostomía atendida en el Centro Especializado de Rehabilitación y verificar la percepción de los usuarios sobre la calidad, seguridad, cantidad y frecuencia de intercambio de los equipos brindados por el servicio. Se trata de un estudio transversal realizado en un Servicio de Atención de Ostomías del interior del estado de São Paulo, Brasil. Se entrevistaron 126 personas con ostomía intestinal entre los meses de abril y junio de 2019, en el día programado para la adquisición del equipo. Entre los participantes del estudio, el $62,90 \%$ eran hombres y el 64,29\% con ostomías permanentes; El 80,16\% dijo estar totalmente satisfecho con la calidad del material recibido y el 67,46\% expresó total seguridad con el uso de los equipos de recolección; El 92,08\% dijo estar satisfecho con la orientación recibida por el equipo de salud y los materiales recibidos. Las pruebas estadísticas no mostraron dependencia entre la calidad del equipo recolector y el resto de variables ( $\mathrm{p}>0.05)$. La mayoría de los encuestados informaron estar satisfechos con la calidad del equipo y la atención brindada por el servicio. Se destaca la importancia de la enfermera de estomaterapia, por ser fundamental en el cuidado de los pacientes con ostomías, desde el preoperatorio y el seguimiento postoperatorio, con indicación del equipo colector adecuado para lograr la rehabilitación y desarrollo de la autonomía del paciente. persona que tiene una ostomía.

Palabras clave: Percepción; Estomia; Atención a la salud; Centros de rehabilitación; Atención al paciente.

\section{Introdução}

A satisfação do usuário com o atendimento do serviço de saúde é um dos resultados mais desejados, pois pode influenciar na adesão dos usuários ao tratamento, consequentemente trazer um desfecho terapêutico positivo com melhora da qualidade de vida dos pacientes e da assistência oferecida pelos profissionais (Bandeira, Damaceno, Weiller, \& Lopes, 2020). São escassos os estudos relacionados à satisfação de pacientes com estomias sobre a assistência recebida. A maioria das pesquisas é direcionada para a qualidade de vida ou complicações pós cirúrgicas.

Muitas pessoas com estomia sofrem com a falta de informação e orientação no contexto hospitalar, tanto no pré operatório como no pós operatório, o que pode comprometer seu processo de reabilitação, influenciar nas relações interpessoais (Sá, Pinheiro, Araújo, Falcão, \& Chagas, 2018) e consequentemente no desenvolvimento de autonomia. Em muitos casos, a pessoa com estomia ou seu cuidador assumem os cuidados específicos relacionados a estomia sem contar com orientação, acompanhamento e suporte de profissionais e serviços destinados para tal fim, deste modo, não é incomum encontrar pessoas utilizando equipamentos coletores de forma inadequada e padecendo de complicações precoces passíveis de prevenção, como por exemplo a dermatite periestomia (Cerqueira et al., 2020).

Segundo a portaria $\mathrm{n}^{\circ} 1.820$ de 13 de agosto de 2009 é direito da pessoa, na rede de serviço de saúde ter atendimento adequado, humanizado, acolhedor, livre de qualquer discriminação, por equipe multiprofissional capacitada e em condições adequadas de atendimento. Cabe ao enfermeiro, utilizando-se do processo de enfermagem, identificar as necessidades individuais da pessoa e planejar a alta hospitalar junto com a equipe multidisciplinar, com o propósito de garantir a continuidade dos cuidados de saúde e o uso eficiente dos recursos da instituição e da comunidade para a desospitalização segura, por meio da referência e contra referência, conforme preconiza a portaria GM/MS nº 3.390 de dezembro de 2013, que institui a Política Nacional de Atenção Hospitalar (PNHOSP) no âmbito do Sistema Único de Saúde (SUS).

São poucos os hospitais/instituições de saúde que possuem o Enfermeiro Estomaterapeuta no seu quadro de profissionais, ou que apresentam algum tipo de protocolo de treinamento para assistência durante o período pré e pós-cirúrgico à pessoa com estomia. Este enfermeiro é qualificado para orientar a pessoa com estomia e sua família, desde o momento do conhecimento da necessidade da estomia até a alta hospitalar e seguimento ambulatorial, orientando e executando ações especializadas com programas educativos sistematizados, incluindo cuidados com a estomia, com pele periestomia e com o 
manuseio dos equipamentos coletores e adjuvantes, o que resultará naturalmente em melhor qualidade de vida deste paciente (Monteiro, Carvalho, Medeiros, Silva, \& Guilhem, 2018).

Assim, surgiram os questionamentos do estudo: Qual a avaliação que os pacientes com estomias fazem sobre o atendimento e os equipamentos fornecidos pelo Centro Especializado de Reabilitação? Os pacientes sentem-se seguros com o uso do e equipamento fornecido? Os pacientes estão satisfeitos com a frequência de troca do equipamento coletor?

Para responder estas questões, os objetivos da pesquisa foram: caracterizar a população que possui estomia atendidos no Centro Especializado de Reabilitação, verificar a percepção dos pacientes com relação a qualidade, segurança, quantidade e a frequência de troca dos equipamentos fornecidos pelo serviço, com a finalidade de aprimorar a assistência oferecida a estas pessoas.

\section{Método}

Trata-se de um estudo exploratório, transversal, do tipo estudo de caso, de natureza quantitativa, conforme preconiza Pereira et al (2018). O estudo foi desenvolvido no Centro Especializado de Reabilitação (CER II), unidade pública de referência, vinculada ao Sistema Único de Saúde (SUS), localizado no interior do estado de São Paulo - Brasil. A Unidade conta com um atendimento multidisciplinar, formado pelo Enfermeiro Estomaterapeuta, Médico Coloproctologista, Nutricionista, Psicóloga e Assistente Social, com o objetivo de oferecer a pessoa estomizada um atendimento integral e de qualidade, desenvolvendo ações de autocuidado, prevenção de complicações e fornecimento de equipamentos coletores/adjuvantes para o desenvolvimento da autonomia enquanto protagonista de sua existência.

A população do estudo foi de 164 pacientes com estomias intestinais cadastrados no serviço para avaliação da estomia pelo enfermeiro estomaterapeuta e aquisição gratuita de equipamentos coletores, agendados para o período entre os meses de abril a junho de 2019. Os critérios de inclusão foram: possuir estomia por um período igual ou superior a seis meses, ter idade igual ou maior de 18 anos, ter capacidade de entendimento e compreensão das instruções para participar da pesquisa.

O critério de exclusão foi não comparecer na unidade para aquisição de equipamentos ou consulta durante o período de coleta dos dados. Assim, foram excluídos 12 pacientes que se recusaram participar do estudo e 26 que o familiar compareceu para adquirir os materiais, apresentando atestado de incapacidade do paciente. Portanto, a amostra foi composta por 126 pessoas que possuiam estomias.

A coleta de dados foi realizada por meio de entrevista, com duração de 15 a 20 minutos e análise de prontuário. A entrevista foi realizada pelo pesquisador no CER II, no dia da aquisição dos equipamentos, em uma sala reservada, seguindo um roteiro com questões objetivas, contendo dados sociodemográficos e clínicos, tipo e quantidade de equipamento coletor recebido do serviço, se os equipamentos ofereciam segurança ou não para uso diário e três questões sobre a satisfação (totalmente satisfeito, parcialmente satisfeito e insatisfeito) com o equipamento fornecido, a frequência da troca do equipamento coletor, e com relação ao atendimento na unidade. Vale salientar que o termo satisfação foi utilizado no intuito de facilitar a compreensão do usuário em relação a sua percepção do serviço prestado e equipamentos fornecidos, como forma de obtenção das respostas e reduzir vieses de entendimento.

A análise do prontuário foi realizada para coleta dos dados clínicos, informações referentes à patologia, data e tipo de procedimento cirúrgico, tipo de estomia, localização da estomia e equipamento coletor indicado. Todas as análises foram obtidas por meio do Software Statistical Package for the Social Sciences (SPSS) Statistics (Versão 23) atreladas às funcionalidades do software Microsoft ${ }^{\circledR}$ Excel (Versão 2016). O perfil da amostra foi realizado de forma descritiva, contemplando as variáveis analisadas e seus desdobramentos. No âmbito inferencial foi traçado como objetivo estatístico a análise de independência e predição entre as variáveis propostas no escopo do trabalho, utilizando dentro dos padrões esperados o teste U de Mann-Whitney e Regressão Linear Multivariada. A significância dos resultados considerou-se os valores de $p<0,05$. 
O Projeto de pesquisa foi aprovado pelo Comitê de Ética em Pesquisa local, conforme Resolução 466/2012, sob Parecer $\mathrm{n}^{\circ}$ 2.748.183/2018 e os participantes assinaram o termo de consentimento livre e esclarecido antes da coleta dos dados.

\section{Resultados}

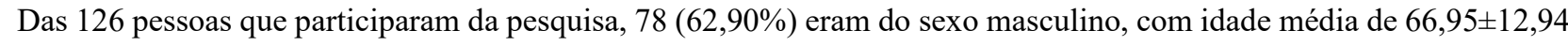
anos, idade mínima de 39 e máxima 91 anos. Em relação a situação conjugal 65 (51,59\%) viviam com companheiro(a), com predomínio de escolaridade de um à cinco anos $(67-53,17 \%) ; 81(64,29 \%)$ referiram atividade laboral remunerada no período anterior à cirurgia e $78(61,90 \%)$ afirmaram estarem aposentados após a cirurgia (Tabela1).

Tabela 1 - Distribuição das características sociodemográficas dos participantes do estudo (n=126). São José do Rio Preto, SP, Brasil, 2019

\begin{tabular}{lllr}
\hline \multicolumn{1}{c}{ Variáveis } & Categorias & $\mathrm{n}(126)$ & $\%$ \\
\hline \multicolumn{1}{c}{ sexo } & masculino & 78 & 61,90 \\
Faixa etária & feminino & 48 & 38,10 \\
& $40-69$ & 70 & 55,56 \\
Situação conjugal & 70 e + & 55 & 43,65 \\
& $18-39$ & 1 & 0,79 \\
Anos que & Com companheiro & 65 & 51,59 \\
frequentou & Sem companheiro & 61 & 48,41 \\
a escola & De 1 á 5 anos & 67 & 53,17 \\
& Acima de 11 anos & 42 & 33,33 \\
Ocupação & De 6 á 10 anos & 10 & 7,94 \\
anterior & Analfabeto & 7 & 5,56 \\
à construção & Trabalho formal & 81 & 64,29 \\
da estomia & Do lar & 23 & 18,25 \\
& Aposentado & 18 & 14,29 \\
Ocupação após & Afastado & 3 & 2,38 \\
a construção & Desempregado & 1 & 0,79 \\
da estomia & Aposentado & 78 & 61,90 \\
& Afastado & 19 & 15,08 \\
& Do lar & 15 & 11,90 \\
& Trabalho formal & 10 & 7,94 \\
& Desempregado & 4 & 3,17 \\
\hline
\end{tabular}

Fonte: Autores (2020).

Como principal causa de confecção da estomia, a neoplasia apresentou maior índice, 79 (62,70\%) pacientes. A Colectomia foi a técnica cirúrgica mais empregada (56 - 44,44\%) e 81 (64,29\%) com estomia de permanência definitiva; 36 $(29,00 \%)$ com tempo de estomia variando entre 60 e 119 meses.

Setenta pacientes $(55,56 \%)$ afirmaram ter recebido as orientações relacionadas a confecção da estomia e 66 (52,38\%) referiram não terem sido orientados no pré-operatório sobre a estomia e cuidados. Sobre orientação de troca correta do equipamento coletor, $106(84,13 \%)$ relataram que receberam orientação e $104(82,54 \%)$ afirmaram receber orientação após a cirurgia sobre cuidados com a estomia e manuseio do equipamento coletor. Em relação ao atendimento realizado pela equipe de saúde do CER II, 111 (88,10\%) pacientes relataram estar totalmente satisfeitos; $113(89,68 \%)$ mencionaram o agendamento de consultas com o médico e Enfermeiro Estomaterapeuta como a maior facilidade no serviço e 95 (75,40\%) não relataram dificuldades. 
O equipamento coletor mais utilizado, mencionado por 90 (71,43\%) entrevistados foi o equipamento coletor de uma peça, sendo que em relação à quantidade, $104(82,54 \%)$ recebiam 10 unidades, 90 (71,43\%) não recebiam nenhum material adjuvante e $56(44,44 \%)$ realizavam a troca do equipamento cada três dias.

Ao serem questionados sobre a quantidade dos equipamentos recebidos serem suficientes para uso mensal, $116(92,06 \%)$ afirmaram ser suficientes, sendo que do total, 119 (94,44\%) referiram nunca terem ficado sem receber o material e $108(85,71 \%)$ relataram que o equipamento atende totalmente às suas necessidades. Em relação à satisfação do equipamento coletor, 101 $(80,16 \%)$ afirmaram estar totalmente satisfeitos com a qualidade do material recebido e $85(67,46 \%)$ referiram estar totalmente seguros com o uso diário do equipamento (Tabela 2).

Tabela 2 - Relatos de satisfação com o equipamento coletor recebido no Centro de Reabilitação (n=126). São José do Rio Preto, SP, Brasil, 2019.

\begin{tabular}{cccc}
\hline Variáveis & Satisfação & $\mathrm{N}$ & $\%$ \\
\hline Equipamento & Totalmente & 108 & $85,71 \%$ \\
& Parcialmente & 18 & $14,29 \%$ \\
& Não & 0 & $0,00 \%$ \\
Qualidade do equipamento coletor & Totalmente & 101 & $80,16 \%$ \\
& Parcialmente & 22 & $17,46 \%$ \\
& Insatisfeito & 3 & $2,38 \%$ \\
Segurança com o equipamento & Totalmente & 85 & $67,46 \%$ \\
& Parcialmente & 34 & $26,98 \%$ \\
& Inseguro & 7 & $5,56 \%$ \\
\hline
\end{tabular}

Fonte: Autores (2020).

Em relação a satisfação com a qualidade do equipamento, três manifestaram insatisfação com a qualidade e 93 (92,08\%) afirmaram estar totalmente satisfeitos. Todos relataram que receberam orientações do serviço (CER II). Com os testes estatísticos foi possível afirmar que não há evidências estatísticas de dependência entre as variáveis satisfação com a qualidade do equipamento coletor e orientação recebida, $\mathrm{p}=0,826$. (Tabela 3 ).

Tabela 3 - Relatos de satisfação com a qualidade do equipamento coletor segundo as orientações recebidas no Centro de Reabilitação (n=126). São José do Rio Preto, SP, Brasil, 2019.

Satisfação com a qualidade do equipamento coletor

Orientações recebidas

Insatisfeito

Parcialmente Satisfeito

Totalmente Satisfeito

Valor $\mathrm{p}$

\begin{tabular}{ccccccc}
\hline & $\mathrm{n}$ & $\%$ & $\mathrm{~N}$ & $\%$ & $\mathrm{~N}$ & $\%$ \\
\hline Não & 0 & 0,00 & 3 & 13,64 & 8 & 7,92 \\
Sim & 3 & 100,00 & 19 & 86,36 & 93 & 92,08 \\
Total & 3 & 100,00 & 22 & 100,00 & 101 & 100,00 \\
\hline
\end{tabular}

Fonte: Autores (2020). 
Com relação à frequência de troca do equipamento coletor, entre os que relataram trocar a cada dois dias (14), 10 $(71,43 \%)$ relataram estar totalmente satisfeitos com a qualidade do equipamento e, entre os que mantém tempo de troca acima de quatro dias (53), 46 (86,79\%) também relataram totalmente satisfeitos. (Tabela 4)

Observou-se que também não há evidências estatísticas de dependência entre as variáveis satisfação e frequência de troca do equipamento (Tabela 4).

Tabela 4 - Relatos de satisfação segundo a frequência de troca do equipamento coletor (n=126) São José do Rio Preto, SP, Brasil, 2019

Frequência de Troca do Equipamento Coletor

\begin{tabular}{cccccccccc} 
Satisfação & \multicolumn{2}{l}{2 Dias } & \multicolumn{2}{c}{3 Dias } & \multicolumn{2}{c}{ Acima de 4} \\
& & \multicolumn{1}{c}{ Dias } & Todos os Dias & Valor p \\
& $\mathbf{n}$ & $\mathbf{\%}$ & $\mathbf{n}$ & $\mathbf{\%}$ & $\mathbf{n}$ & $\mathbf{\%}$ & $\mathbf{n}$ & $\mathbf{\%}$ & 0,509 \\
\hline $\begin{array}{c}\text { Insatisfeito } \\
\text { Parcialmente }\end{array}$ & 0 & 0,00 & 1 & 1,79 & 2 & 3,77 & 0 & 0,00 & \\
$\quad$ Satisfeito & 4 & 28,57 & 13 & 23,21 & 5 & 9,43 & 0 & 0,00 & \\
Totalmente Satisfeito & 10 & 71,43 & 42 & 75,00 & 46 & 86,79 & 3 & 100,00 & \\
$\quad$ Total & 14 & 100,00 & 56 & 100,00 & 53 & 100,00 & 3 & 100,00 & \\
\hline
\end{tabular}

Fonte: Autores (2020).

Entre aqueles que estão totalmente satisfeitos, 80 (79,21\%) afirmaram totalmente seguros com o uso diário do equipamento coletor e com o teste estatístico constatou-se que não há evidências estatísticas de dependência entre elas, $\mathrm{p}=0,330$.

Dois $(66,67 \%)$ dos insatisfeitos com o equipamento coletor apresentavam colostomia e 75 (72,73\%) dos totalmente satisfeitos também apresentam o mesmo tipo de estomia. Ao realizar o teste estatístico entre estas variáveis foi possível observar que não há evidências estatísticas de dependência, p=0,898.

Trinta $(29,70 \%)$ dos que relataram totalmente satisfeitos com a qualidade do equipamento coletor apresentavam tempo com a estomia de 60 a 119 meses. Constatou-se também que não há evidências de dependência entre elas $(\mathrm{p}=0,456)$.

\section{Discussão}

O presente estudo mostrou predominância no sexo masculino, com média de idade 66,95 anos; vivem com companheira e frequentaram a escola de um a cinco anos. Este achado vai ao encontro de outro estudo realizado com pacientes com estomia atendidos no departamento de Órtese e Prótese, no estado do Paraná, que mostrou também uma população com a maioria do sexo masculino, com idade média de 62,44 anos, casada, e ensino fundamental incompleto (Sousa, Rosa, Rinaldi, \& Campos, 2020). Diferente do estudo realizado em 2014, que avaliou a satisfação com o uso de equipamentos coletores de pessoas cadastradas no Programa de Assistência as Pessoas com Estomia, em uma cidade no sul de Minas Gerais, onde o predomínio foi de mulheres casadas, com média de idade de 61,60 anos (Spenazato, Santos, Morita, \& Paula, 2016).

Outros estudos relatam que o medo da detecção precoce de doenças, associado com o temor do diagnóstico são fatores que podem justificar o menor acesso aos serviços de saúde pelos homens, contribuindo para procura de recursos alternativos, como a automedicação, uso da medicina popular e ervas medicinais caseiras, considerados por eles como eficaz no tratamento de algum tipo de agravo, o que os torna mais vulneráveis a complicações e diagnósticos em estágios avançados da doença, como 
as neoplasias colorretais, cujo o tratamento é cirúrgico com confeção de estomia (Oliveira, Daher, Silva \& Andrade, 2015; Teixeira \& Cruz, 2016).

O nível de escolaridade da população em estudo apresenta predomínio de indivíduos com ensino fundamental, assemelhando-se a estudos em diferentes localidades brasileiras (Sousa, Rosa, Rinaldi, \& Campos, 2020; Miranda, Luz, Sonobe, Andrade, \& Moura, 2016; Capilla-Díaz et al, 2019)

Com relação à atividade laboral dos pacientes com estomia, estudos apontam que muitos pacientes preferem se afastar ou se isolar para evitar constrangimentos perante a sociedade, devido a ruídos e odores, ou insegurança pela possibilidade de ocorrer acidentes com o equipamento coletor de efluentes, como vazamento de fezes; o que pode muitas vezes dificultar a própria aceitação no processo de readaptação a sua nova condição com estomia (Nascimento, Trindade, Luz \& Santiago, 2011; Faria, Labre, Sousa \& Almeida, 2018; Capilla-Díaz et al, 2019).

Outro aspecto a ser considerado para o afastamento do trabalho é a possibilidade da utilização dos direitos da legislação regulamentada pelo Decreto $\mathrm{n}^{\mathrm{0}} 5.296$, de 02 de dezembro de 2004, que estabelece que qualquer pessoa que apresente alteração completa ou parcial de um ou mais segmentos do corpo humano que resulte em comprometimento da função orgânica, no qual inclui a pessoa com estomia, pode receber auxílio do governo durante o tratamento da doença ou permanentemente.

Um dos princípios fundamentais no cuidado a pessoa com estomia de eliminação é utilizar um equipamento coletor de efluentes com aderência segura e consistente, que se adeque tanto as características da estomia como da área periestomia, a fim de não apresentar vazamentos desde a sua colocação até sua retirada. Quando a adaptação é inadequada, vários problemas adicionais podem ocorrer, como insegurança na manutenção do equipamento, odor, vazamentos, maior tempo dispensado com os cuidados com a estomia, sujidade das roupas, estigma e problemas sociais, como constrangimento e participação limitada em atividades interpessoais e sociais (Colwell et al, 2019). O contato da pele com o efluente pode causar dermatite na pele periestomia, o que prejudica ainda mais a permanência do equipamento coletor, determina a implementação de cuidados adicionais como o uso de adjuvantes para melhor aderência do equipamento (Santos \& Cesaretti., 2015).

Uma revisão de literatura que abordou aspectos psicológicos dos pacientes com estomias destaca que o plano de assistência a estes pacientes deve contemplar, desde o período pré operatório, os aspectos emocionais, sociais, culturais e espirituais, com a finalidade de contribuir para a confiança no uso do equipamento e melhor reabilitação física e psicossocial (Silva, Santos, Rosado, Galvão, Sonobe, 2017).

Estes fatores destacam a importância do atendimento e acompanhamento multidisciplinar à pessoa com estomia de forma longitudinal, iniciando já no pré-operatório e perdurando pelo período que a pessoa permanecer com a estomia. Neste sentido se faz necessário uma organização robusta dos serviços que compõem a rede de atendimento, com definição de fluxos, itinerário terapêutico, protocolos clínicos e sistema de regulação de referência e contrarreferência que possibilitem o segmento desses usuários (Portaria $\mathrm{n}^{\circ}$ 793, 2012). Isto pode refletir de forma positiva na satisfação e no processo de adaptação com a estomia, conforme preconiza a portaria 3.390 PNHOSP/2013, que diz respeito a desospitalização e alta responsável. Implica também no acompanhamento periódico do paciente por profissionais capacitados, o que está diretamente relacionado a reabilitação, desenvolvimento de autonomia e melhoria na qualidade de vida da pessoa com estomia (Finlay, Sexton \& McDonald, 2018).

A reinserção do indivíduo no ambiente de trabalho e a convivência com outras pessoas contribuem de forma positiva para seu bem-estar, contudo, é essencial que os profissionais de saúde estejam aptos para informar sobre leis trabalhistas, bem como a participação no processo de readaptação à sua nova condição de vida, o manuseio dos equipamentos coletores e possíveis intercorrências, para que a pessoa com estomia se sinta segura para retornar às atividades sociais e laborais (Selau et al, 2019).

Com o objetivo de garantir um atendimento integral e especializado às pessoas com estomia, o Ministério da Saúde publicou a Portaria MS/GM no. 400 (2009), que estabelece as Diretrizes Nacionais para a Atenção à Saúde das pessoas com 
estomias no âmbito do SUS, a serem observadas em todas as unidades federadas, respeitadas as competências das três esferas de gestão. Esta portaria também define que a atenção à saúde das pessoas com estomias seja composta por ações desenvolvidas na atenção básica, com orientações para o autocuidado e prevenção de complicações, além de ações desenvolvidas nos Serviços de Atenção à Saúde das Pessoas com Estomia. Esse serviço deve ser composto por uma equipe multidisciplinar, à qual compete o planejamento da assistência às pessoas com estomias, contando com a participação do próprio usuário e seus familiares, com a finalidade de garantir a adesão e o seguimento do cuidado com qualidade no domicílio (Comes et al, 2016).

Sobre o tempo de estomia, motivo e procedimento cirúrgico abordado, pode-se observar semelhanças com o estudo realizado com os pacientes registrados no Centro de Assistência de Alta Complexidade em Oncologia (CACON), em 2013, no Hospital Universitário e na Unidade de Pronto Atendimento em Maceió, onde foram encontrados como motivo da estomia o câncer colorretal $(48,60 \%)$, que resultaram em colostomia $(84,60 \%)$ e $43,00 \%$ de permanência definitiva (Neto, Fernandes \& Didoné, 2016).

A maioria dos participantes declararam totalmente satisfeitos com o serviço e atendimento do enfermeiro estomaterapeuta. Um estudo qualitativo realizado na Espanha ressaltou que as pessoas que possuem estomia consideram fundamental a relação com os profissionais da saúde para o enfrentamento da sua nova condição com segurança e sem medo do retorno à vida cotidiana, com autossuficiência, e destacam que as dificuldades encontradas s é a falta de profissionais treinados para fornecer cuidados com a estomia, o que traz sentimento de insegurança e desamparo. Karaca e Durna (2019) ainda destacam que tanto nas instituições privadas como na Atenção Primária à Saúde há escassez de enfermeiros estomaterapeutas.

Com relação às orientações sobre cuidados com a estomia e troca de equipamento coletor podemos observar divergências com o estudo de Spenazato et al. (2016) onde 81,25\% dos entrevistados relataram não ter recebido orientação no pré operatório e $62,50 \%$ relataram não ter recebido orientação no pós operatório, porém todos afirmaram ter recebido orientação no ambulatório de estomaterapia, tanto em relação aos cuidados com a estomia como no manuseio de equipamentos, e também relataram satisfação com os equipamentos fornecidos, os quais $53,10 \%$ faziam uso de equipamento de duas peças. $O$ mesmo estudo identificou que a maioria dos pacientes apresentou um nível de satisfação "bom" com os materiais fornecidos, como o equipamento coletor, aderência do equipamento à pele e a segurança no fechamento do mesmo, facilidade na higienização, tempo de troca de quatro a seis dias, mas destacou que a maioria deles não recebeu orientação no pré-operatório (Spenazato, et al, 2016).

Um estudo realizado em um hospital na Turquia, desenvolvido com pacientes internados para tratamento clínico verificou satisfação dos pacientes com os cuidados de enfermagem em geral, mas pouca satisfação com as informações fornecidas pela equipe de enfermagem; os pacientes relataram que gostariam de receber informações sobre sua doença, estado de saúde e prognósticos de sua condição de saúde. Miranda, Carvalho e Paz (2018) destacam que as habilidades de comunicação dos profissionais de saúde desempenham um papel fundamental para garantir que os pacientes se sintam valorizados e bem cuidados; concluíram que os gerentes deveriam incluir no programa de treinamento as habilidades e atitudes dos enfermeiros assistenciais segundo as expectativas dos pacientes, para assegurar a satisfação e a melhor qualidade dos cuidados de enfermagem oferecidos.

Para Freire et al (2017), o enfermeiro é facilitador no processo de autoaceitação da nova condição de vida do paciente que tem uma estomia, o que pode estar diretamente relacionado ao processo de implementação de ações que favoreçam uma assistência direcionada e satisfatória para a pessoa em particular. O enfermeiro tem papel fundamental no apoio emocional e psicológico para os pacientes e familiares em todos os momentos do tratamento desde a informação do diagnóstico, como na segurança dos cuidados e orientações para a alta hospitalar, sempre com conhecimentos, atitudes e habilidades profissionais qualificadas (Buchanan, Dawkins \& Lindo, 2015; Goh, Ang, Chan, He, \& Vehvilainen-Julkunen, 2016).

Os estudos que avaliam a satisfação do paciente com os serviços de saúde têm o objetivo de compreender as expectativas e sugestões dos usuários buscando constantemente a melhoria do serviço, os quais poderão fornecer dados para o ajuste de desempenho dos serviços oferecidos e melhoria da experiência do usuário. 
Por esse motivo, acredita-se que a percepção do usuário em relação ao serviço deve ser constantemente investigada, para tanto deve-se buscar estabelecer canais e formas de comunicação que possibilitem identificar variáveis que afetam o atendimento e determinar quais intervenções devem ser priorizados com base nas respostas dos pacientes (Worku \& Loha, 2017).

As limitações do estudo foram: dificuldades na realização da coleta de dados devido ao índice de abstenção dos pacientes em consulta durante o período de coleta dos dados, relacionado com o agendamento trimestral de retorno médico, a quantidade limitada de estudos relacionados à satisfação do paciente com estomia com relação ao atendimento recebido e a ausência de instrumento de coleta validado e especifico para a avaliação da satisfação da pessoa com estomia em relação aos equipamentos coletores e adjuvantes. Os instrumentos encontrados na literatura avaliam qualidade de vida e grau de implantação da estrutura e de processo dos Serviços de Atenção à Saúde da Pessoa Estomizada.

\section{Conclusão}

Este estudo apresenta informações sobre a percepção da pessoa com estomia tanto em relação ao atendimento profissional como o tipo de equipamento fornecido. Em geral, embora não haja significância estatística, os relatos dos usuários revelam avaliação positiva tanto em relação aos profissionais como em relação aos materiais fornecidos. A facilidade de acesso as consultas com os profissionais favorecem a construção da relação terapêutica entre o profisssional e o usuário do serviço, o que pode facilitar a identificação precoce de situações que requeiram intervenção da equipe, com impacto na percepção do usuário em relação ao trabalho da equipe.

Cabe destacar o papel da consulta de enfermagem de forma individualizada, capacitando cuidadores, incentivando o autocuidado e a reabilitação para melhoria da qualidade de vida e desenvolvimento da autonomia do paciente. Conforme apresentado anteriormente, nem todas as pessoas com estomias tem orientações durante a internação, muitos deixam a instituição hospitalar sem preparo para cuidar da estomia, neste contexto de cuidados é imprescindível que o enfermeiro tenha conhecimento, infraestrutura e equipamentos para realizar a consulta de enfermagem de forma holística e individualizada.

O estudo contribui para reflexão do enfermeiro a respeito da importância do seu papel no processo das práticas de cuidado ao paciente com estomia e é um ponto de partida para novos estudos, incluindo pesquisas qualitativas sobre o tema.

Sugere-se estudos voltados para a compreensão das principais necessidades do paciente com estomia no atendimento recebido nas unidades e instituições de saúde.

\section{Referências}

Bandeira, D., Damaceno, A. N., Weiller, T. H., \& Lopes, L. F. D. (2020) Avaliação da coordenação da atenção por usuários dos serviços de atenção primária à saúde. Rev Min Enferm., 24,e-1278. http://www.dx.doi.org/10.5935/1415-2762.20200007

Capilla-Díaz, C., Bonill-de Las Nieves, C., Hernández-Zambrano, S. M., Montoya-Juárez, R., Morales-Asencio, J, M., Pérez-Marfil, M. N., \& Hueso-Montoro, C. (2019). Living with an Intestinal stoma: A qualitative Systematic Review Concepcion. Qualitative Health Research [Internet], $29(9)$, 1255-1265. https://doi.org/10.1177/1049732318820933.

Cerqueira, L. C. N., Cacholi, S. A. B., Nascimento, V. S., Koeppe, G. B. O., Torres, V. C. P., \& Oliveira, P. P. (2020). Clinical and sociodemographic characterization of ostomized patients treated at a referral center. Rev Rene, 21, e42145. http://dx.doi.org/10.15253/2175-6783.20202142145

Colwell, J. C., Bain, K. A., Hansen, A. S., Droste, W., Vendelbo, G., \& James-Reid, S. (2019). International Consensus Results. Development of Practice Guidelines for Assessment of Peristomal Body and Stoma Profiles, Patient Engagement, and Patient Follow-up. J Wound Ostomy Continence Nurs, 46(6), 497504. https//doi.org/10.1097/WON.0000000000000599

Comes, Y., Trindade, J. S., Shimizu, H. E., Hamann, E. M., Bargioni, F., \& Ramirez, L., Sanchez, M. N., \& Santos, L. M. P. (2016). Avaliação da satisfação dos usuários e da responsividade dos serviços em municípios inscritos no Programa Mais Médicos. Ciênc. saúde coletiva, 21(9), 2749-2759 http://dx.doi.org/10.1590/1413-81232015219.16202016.

Decreto n. 5.296 de 02 de Dezembro de 2004. Estabelece normas gerais e critérios básicos para a promoção da acessibilidade das pessoas portadoras de deficiência ou com mobilidade reduzida, e dá outras providências. http://www.planalto.gov.br/ccivil_03/_ato2004-2006/2004/decreto/d5296.htm

Faria, F. L., Labre, M. M., Sousa, I. F. \& Almeida, R. J. (2018) Avaliação da qualidade de vida em pacientes com estomia intestinal. Arq. Ciênc. Saúde, 25(2), 08-14. https://doi.org/10.17696/2318-3691.25.2.2018.924 
Finlay, B., Sexton, H. \& McDonald, C. (2018). Care of patients with stomas in general practice. Aust J Gen Pract. 47(6), $362-365$. https://doi.org/10.31128/AJGP-12-17-4430

Freire, D. A., Angelim, R. C. M., Souza, N. R., Brandão, B. M. G. M., Torres, K. M. S. \& Serrano, S. Q. (2017). Self-image and self-care in the experience of ostomy patients: the nursing look. Rev Min Enferm, 21, e-1019. http://www.dx.doi.org/10.5935/1415-2762.2017002

Goh, M. L., Ang, E. N. K., Chan, Y. H., He, H. G. \& Vehvilainen-Julkunen, K. (2016). A descriptive quantitative study on multi ethnic patient satisfaction with nursing care measured by the revised humane caring scale. Appl Nurs Res, 31,126-131. https//doi.org/10.1016/j.apnr.2016.02.002

Karaca, A. \& Durna, Z. (2019). Patient satisfaction with the quality of nursing care. Nurs Open, 6(2), 535-545. https//doi.org/10.1002/nop2.237

Miranda, L. S. G., Carvalho, A. A. S. \& Paz, E. P. A. (2018) Qualidade de vida da pessoa estomizada: relação com os cuidados prestados na consulta de enfermagem de estomaterapia. Esc Anna Nery, 22(4), e20180075. https//doi.org/10.1590/2177-9465-EAN-2018-0075.

Miranda, S. M., Luz, M. H. B. A., Sonobe, H. M., Andrade, E. M. L. R. \& Moura, ECC. (2016). Caracterização Sociodemográfica e Clínica de Pessoas com Estomia em Teresina. Rev Estima, 14(1), 29-35. https://www.revistaestima.com.br/estima/article/view/117

Monteiro, S. N. C., Carvalho, E. M. P., Medeiros, L., Silva, A. L. \& Guilhem, D. (2018). Educação em saúde para crianças com estomias intestinais: o enfermeiro como mediador do cuidar. Revista pesquisa qualitativa (SP), 6 (10), 44-59. https://doi.org/10.33361/RPQ.2018.v.6.n.10.205

Neto, M. A. F. L., Fernandes, D. O. A. \& Didoné, E. L. (2016). Epidemiological characterization of ostomized patients attended in referral Center from the city of Maceió, Alagoas, Brazil. J. Coloproctol, 36(2), 64-68. https://doi.org/10.1016/j.jcol.2014.08.016

Oliveira, M. M., Daher, D. V., Silva, J. L. L. \& Andrade, S. S. C. A. (2015). Men's health in question: seeking assistance in primary health care. Ciência \& Saúde Coletiva, 20 (1), 273-278. https://doi.org/10.1590/1413-81232014201.21732013

Pereira, A. S., Shitsuka, D. M., Parreira, F. J. \& Shitsuka, R. (2018). Metodologia de pesquisa Científica. [free e-book] Santa Maria/RS. Ed. UAB/NTE/UFSM.

Portaria MS/GM nº.400 de 16 de novembro de 2009. Estabelece Diretrizes Nacionais para a Atenção à Saúde das Pessoas Ostomizadas no âmbito do Sistema único de Saúde - SUS, a serem observadas em todas a unidades federadas, respeitadas as competências das três esferas de gestão. https://bvsms.saude.gov.br/bvs/saudelegis/sas/2009/prt0400_16_11_2009.html

Portaria $\mathrm{n}^{\mathrm{o}}$ 1.820, de 13 de agosto de 2009. Dispõe sobre os direitos e deveres dos usuários da saúde. https://bvsms.saude.gov.br/bvs/saudelegis/gm/2009/prt1820_13_08_2009.html.

Portaria n ${ }^{\circ}$ 3.390, de dezembro de 2013. Institui a Política Nacional de Atenção Hospitalar (PNHOSP) no âmbito do Sistema Único de Saúde (SUS), estabelecendo-se as diretrizes para a organização do componente hospitalar da Rede de Atenção à Saúde (RAS). $<$ http://bvsms.saude.gov.br/bvs/saudelegis/gm/2013/prt3390_30_12_2013.html>.

Portaria n ${ }^{\circ}$ 793, de 24 de abril de 2012. Institui a Rede de Cuidados à Pessoa com Deficiência no âmbito do Sistema Único de Saúde. Diário Oficial da União; Poder Executivo. http://www.anamt.org.br/site/upload_arquivos/legislacao_-_leis_2012_171220131649115795186.pdf

Sá, D. L. F., Pinheiro, R. X., Araújo, M. M. O., Falcão, P. C. \& Chagas, M. X. (2018) Cuidados com o paciente pré cirúrgico de ostomia. Revista Científica Multidisciplinar Núcleo do Conhecimento, 4 (1), 21-30. https://www.nucleodoconhecimento.com.br/saude/paciente-pre-cirurgico-de-ostomia

Santos, V. L. C. G. \& Cesaretti, I. U. R. (2015). Assistência em estomaterapia - cuidando de pessoas com estomia. Atheneu.

Selau, C. M., Limberger, L. B., Silva, M. E. N., Pereira, A. D., Oliveira, F. S. \& Margutti, K. M. M. (2019) Percepção dos pacientes com estomia intestinal em relação às mudanças nutricionais e estilo de vida. Texto Contexto Enferm. http://dx.doi.org/10.1590/1980-265X-TCE-2018-0156

Silva, N. M., Santos, M. A., Rosado, S. R., Galvão, C. M. \& Sonobe, H. M. (2017). Psychological aspects of patients with intestinal stoma: integrative review. Rev. Latino-Am. Enfermagem., 25, e2950. http://dx.doi.org/10.1590/1518-8345.2231.2950

Sousa, J. A. V., Rosa, M. M., Rinaldi, E. C. A. \& Campos, C. G. P. (2020). Perfil de usuários estomizados atendidos em um departamento de órteses e próteses. Enferm. Foco, 11(4), 35-40. https://doi.org/10.21675/2357-707X.2020.v11.n4.2919

Spenazato, R. V. S., Santos, C. R. S., Morita, A. B. P. S. \& Paula, M. A. B. (2016). Satisfação de Pacientes com estomias intestinais quanto ao uso de equipamentos Rev Estima, 14(4), 193-202. https://www.revistaestima.com.br/index.php/estima/article/view/432/pdf

Teixeira, D. B. S. \& Cruz, S. P. L. (2016) Atenção à saúde do homem: análise da sua resistência na procura dos serviços de saúde. Rev Cubana Enferm, 32(4), http://www.revenfermeria.sld.cu/index.php/enf/article/view/985

Worku, M. \& Loha, E. (2017). Assessment of client satisfaction on emergency department services in Hawassa University Referral Hospital, Hawassa, Southern Ethiopia. BMC Emergency Medicine, 17,21. https://doi.org/10.1186/s12873-017-0132-7. 